\title{
Cambio organizacional: análisis de factores sociodemográficos
}

\author{
Esther Julia Castaño González*, Eliana Quiroz González**, Laura Marcela Pulgarín Valencia***, \\ Valentina Britto Cañas****
}

DOI: https://doi.org/:10.33571/revistaluciernaga.v12n24a5

\section{Resumen}

El objetivo de esta investigación fue determinar la relación de la disposición al cambio organizacional con factores sociodemográficos como edad y nivel de escolaridad, al igual que con la diferencia entre sexo masculino y femenino, en una organización de la ciudad de Pereira. Este estudio no experimental, de tipo cuantitativo y correlacional, contó con una muestra de 61 personas $(\mathrm{M}=29$ y $\mathrm{H}=32)$, cuya edad promedio osciló entre 22 y 65 años. Los resultados indican que no hay relación entre la disposición al cambio organizacional con la edad y el nivel de escolaridad. Además, los hombres obtuvieron una mayor puntuación en la dimensión individual $(M=4.7, D E=0.7)$, y las mujeres una mayor puntuación en la dimensión grupal $(M=4.4, D E=0.8)$ y organizacional $(M=4.7, D E=0.8)$.

Palabras clave: Cambio; clima organizacional; comunicación; organizaciones; Pereira.

Recibido. Junio 26,2020 Aceptado. Julio 07, 2020

*Comunicadora Social - Periodista, Especialista en Comunicación Organizacional, Magister en Educación: Desarrollo Humano, Doctora en Relaciones Internacionales Iberoamericanas, Docente Universidad Católica de Pereira. Programa Comunicación Social - Periodismo. Orcid: https://orcid.org/0000-0003-3240-896X; e-mail: julia.castano@ucp.edu.co

**Magíster en Gerencia del Talento Humano, especialista en Gerencia del Talento Humano, Psicóloga. Docente Universidad Católica de Pereira, Programa de Psicología. Orcid: https://orcid.org/0000-0001-9061-8864; e-mail: eliana.quiroz@ucp.edu.co

***Psicóloga, Universidad Católica de Pereira. Orcid: https://orcid.org/0000-0002-9362-807X; e-mail: laura1. pulgarin@ucp.edu.co

****Psicóloga, Universidad Católica de Pereira. Orcid: https://orcid.org/0000-0003-2017-9661; e-mail: vlentina.britto@ucp.udu.co

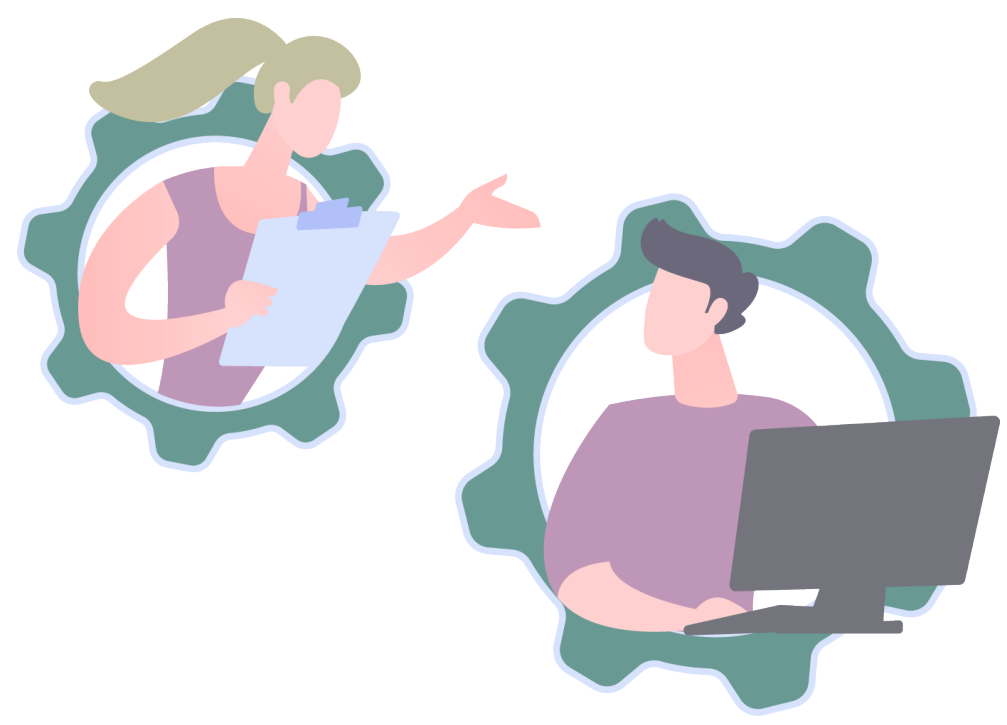




\title{
Disposition to organizational change: analysis of the role of different sociodemographic factors
}

\author{
Esther Julia Castaño González*, Eliana Quiroz González**, Laura Marcela Pulgarín Valencia***, \\ Valentina Britto Cañas ${ }^{* * * *}$
}

DOI: https://doi.org/: 10.33571/revistaluciernaga.v12n24a5

\section{Abstract}

The objective of this research was to determine the relationship between the willingness to organizational change and sociodemographic factors such as age and level of schooling, as well as the difference between male and female gender, in an organization in the city of Pereira. This non-experimental, quantitative, and correlational study had a sample of 61 people $(\mathrm{M}=29$ and $\mathrm{H}=32)$, whose average age ranged between 22 and 65 years. The results indicate that there is no relationship between willingness to organizational change with age and level of schooling. In addition, men scored higher on the individual dimension $(M=4.7, S D=0.7)$, and women scored higher on the group dimension $(M=4.4, S D=0.8)$ and organizational dimension $(\mathrm{M}=4.7, \mathrm{SD}=0.8)$.

Keywords: Disposition; change; organizational climate; age; schooling; sex.

Received. June 26, $2020 \quad$ Accepted. July 7, 2020

*Social Communicator - Journalist, Specialist in Organizational Communication, Master in Education: Human Development, PhD in Iberoamerican International Relations, Professor at Universidad Católica de Pereira. Social Communication - Journalism Program. Orcid: https://orcid.org/0000-0003-3240-896X; e-mail: julia. castano@ucp.edu.co

${ }^{* *}$ Master in Human Talent Management, specialist in Human Talent Management, Psychologist. Professor at Universidad Católica de Pereira, Psychology Program. Orcid: https://orcid.org/0000-0001-9061-8864; e-mail: eliana.quiroz@ucp.edu.co

${ }^{* * * P}$ sychologist, Universidad Católica de Pereira. Orcid: https://orcid.org/0000-0002-9362-807X; e-mail: laura1. pulgarin@ucp.edu.co

****Psychologist, Universidad Católica de Pereira. Orcid: https://orcid.org/0000-0003-2017-9661; e-mail: vlentina.britto@ucp.udu.co

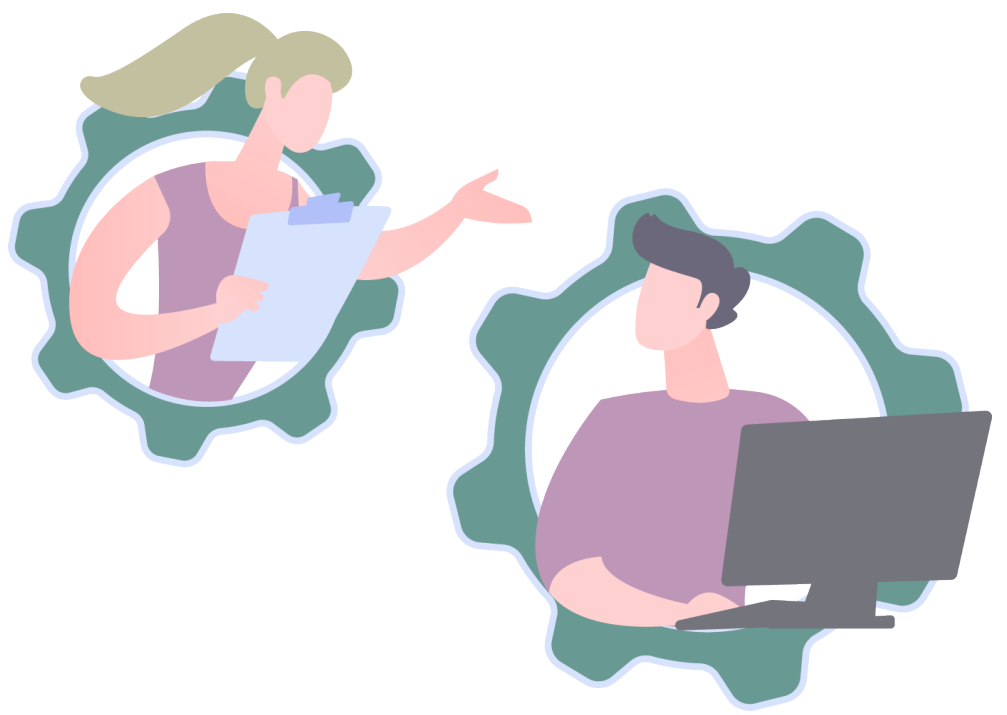




\title{
Mudança organizacional: análise de factores sócio- demográficos
}

\author{
Esther Julia Castaño González*, Eliana Quiroz González**, Laura Marcela Pulgarín Valencia***, \\ Valentina Britto Cañas ${ }^{* * *}$
}

DOI: https://doi.org/: 10.33571/revistaluciernaga.v12n24a5

\section{Resumo}

O objetivo desta investigação era determinar a relação da disposição para a mudança organizacional com fatores sociodemográficos como a idade e o nível de escolaridade, bem como com a diferença entre o sexo masculino e feminino, numa organização da cidade de Pereira. Este estudo não experimental, de tipo qualitativo e correlacional, teve uma amostra de 61 pessoas $(\mathrm{M}=29$ e $\mathrm{H}=32)$, cuja idade média variou entre 22 e 65 anos.

Os resultados indicam que não há relação entre a vontade de mudança organizacional com a idade e o nível de escolaridade. Além disso, os homens tiveram pontuações mais elevadas na dimensão individual. ( $\mathrm{M}=4,7, \mathrm{DE}=0,7)$, e as mulheres tiveram pontuações mais elevadas na dimensão grupal $(M=4,4, D E=0,8)$ e na dimensão organizacional $(M=4,7, D E=0,8)$.

Palavras chave: Mudança; clima organizacional; comunicação; organizações; Pereira.

Recebido. Junho 26, 2020

Aceitado. Julho 07, 2020

*Comunicadora Social - Jornalista, Especialista em Comunicação Organizacional, Mestre em Educação: Desenvolvimento Humano, PhD em Relações Internacionais Ibero-Americanas, Professor na Universidade Católica de Pereira. Comunicação Social - Programa de Jornalismo. Orcid: https://orcid.org/0000-0003-3240896X; e-mail: julia.castano@ucp.edu.co

**Mestre em Gestão de Talentos Humanos, especialista em Gestão de Talentos Humanos, Psicólogo. Professor na Universidade Católica de Pereira, Programa de Psicologia. Orcid: https://orcid.org/0000-0001-9061-8864; e-mail: eliana.quiroz@ucp.edu.co

***Psicóloga, Universidade Católica de Pereira. Orcid: https://orcid.org/0000-0002-9362-807X; e-mail: laura1. pulgarin@ucp.edu.co

****Psicóloga, Universidade Católica de Pereira. Orcid: https://orcid.org/0000-0003-2017-9661; e-mail: vlentina. britto@ucp.udu.co

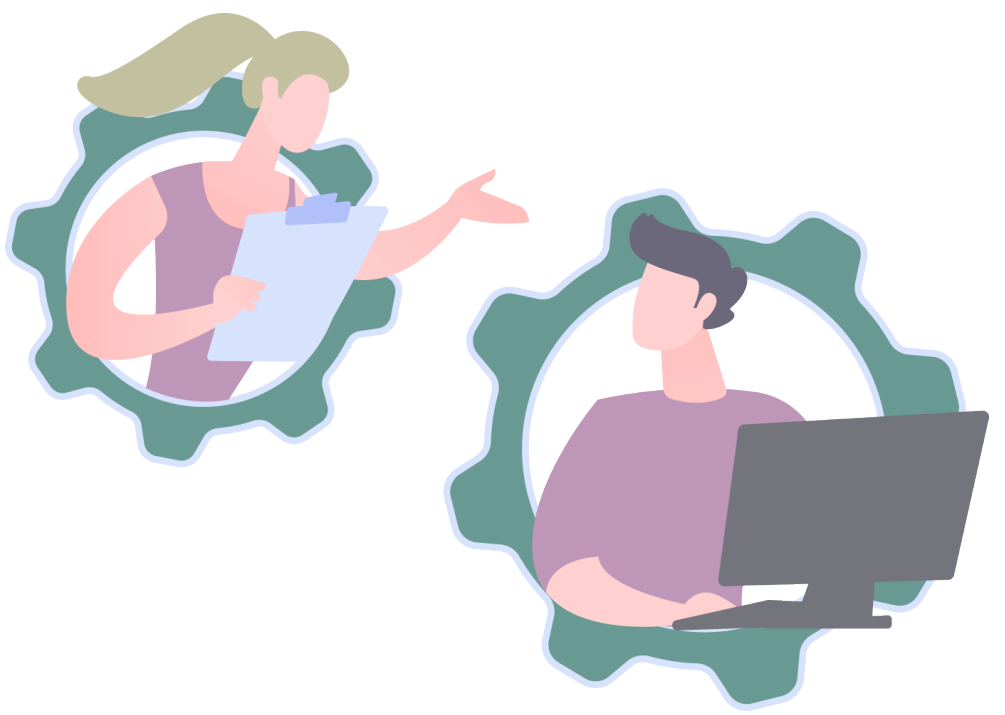




\section{Autoras}

\section{Esther Julia Castaño González} Eliana Quiroz-González

\section{Laura Marcela Pulgarín Valencia} Valentina Britto Cañas

\section{Cambio organizacional:} análisis de factores sociodemográficos.

DOI: https://doi.org/:10.33571/revistaluciernaga.v12n24a5

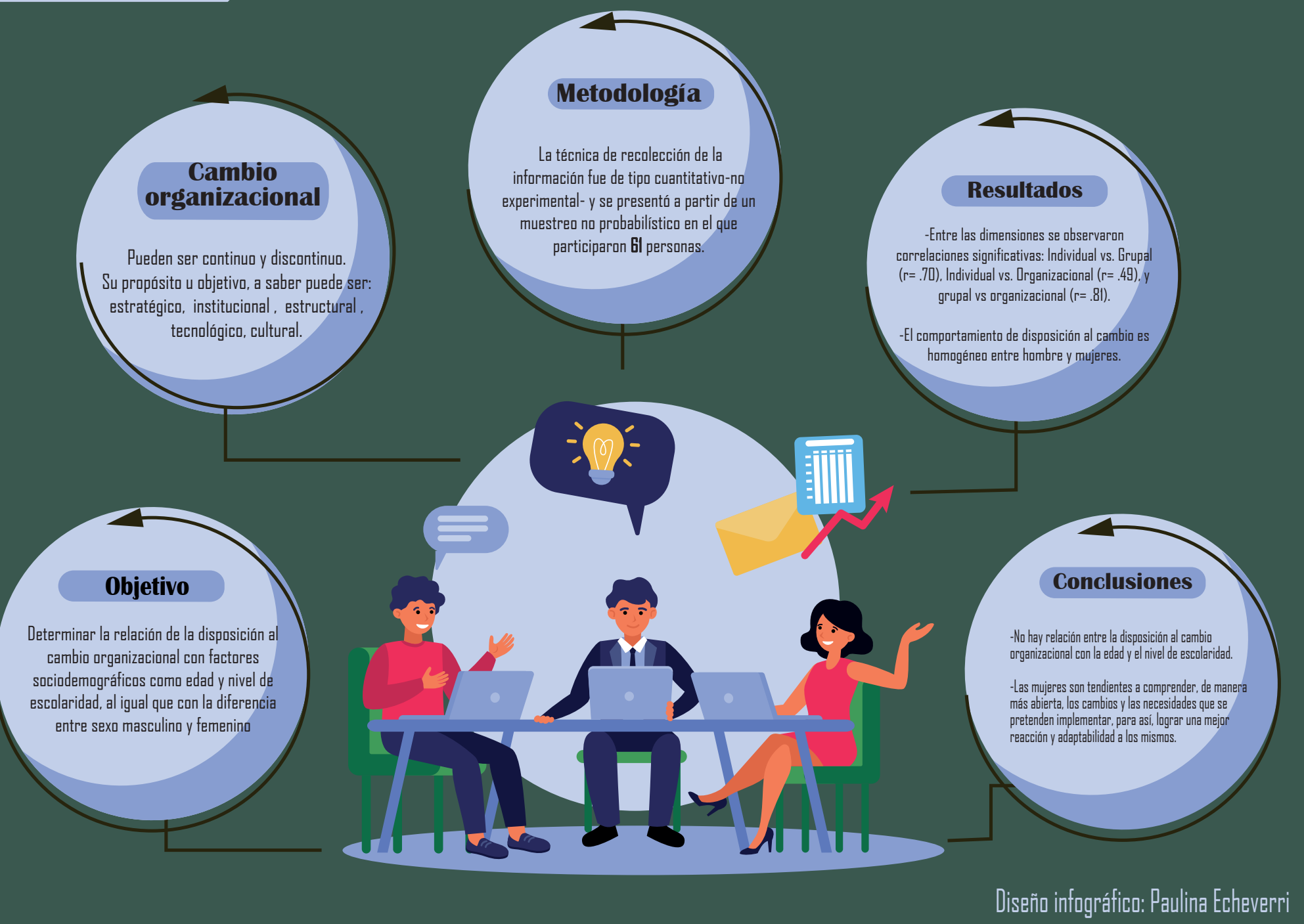




\section{Introducción}

El cambio organizacional representa, para una compañía, su capacidad de adaptación frente a exigencias internas y externas; las internas provienen del cambio de estructura y de comportamientos; las externas, hacen referencia a nuevas tecnologías, cambios políticos, económicos, entre otras demandas que determinan la supervivencia en un mundo empresarial cada vez más competitivo (García, 2011 y Suárez, 2014).

Cabe mencionar que, los procesos de globalización dados en el siglo XXI han obligado a las organizaciones a adaptarse a las exigencias del entorno y a los diferentes y vertiginosos cambios para el logro de sus objetivos. En este sentido, las áreas de recursos humanos cumplen un rol protagónico, pues, desde una perspectiva contingente, estas áreas o departamentos deben diseñar e implementar una amplia gama de prácticas organizacionales adaptadas a los cambios propuestos a nivel corporativo, los cuales deben ser efectuados, preferiblemente, antes de que se coloque en marcha el cambio (Raeder, 2019).

Tal como lo afirma Gutiérrez (2019), cualquier "entidad existe por un propósito y se adapta para dar alcance a un objetivo o estado final deseado, para el cual toma acciones de forma individual o en interacción con otros" (p. 4) y, en este proceso de adaptación surge el cambio organizacional. Ahora bien, para que el cambio sea exitoso, es necesario que se gestione el clima organizacional (Maldonado, Maldonado y Bustamante, 2006; Segredo, 2013), en consecuencia, un clima organizacional sólido es un factor clave para que los cambios se efectúen exitosamente. Así mismo, cuando hay excelencia en la seguridad laboral, se llevan a cabo con satisfacción los cambios organizacionales (Ciro, 2015).

\section{Cambio organizacional}

El cambio organizacional es definido como una respuesta ante exigencias o demandas externas con un rediseño o acomodación interna, la cual busca modificar, transformar o implementar nuevas estrategias para conseguir mejores resultados e incrementar la efectividad empresarial (Acosta, 2002).

El origen de un cambio organizacional generalmente se da en el entorno, que genera, a su vez, decisiones de la organización, por tanto, el cambio organizacional es definido como una modificación de alguna situación, estado o condición; es una transformación de ciertas características (Hernández, Gallarzo y Espinoza, 2011). Por ello, los cambios organizacionales pueden ser experimentados inmediatamente o a largo plazo y afectan directa o indirectamente a todos los individuos de una organización.

Nadler y Tushman (1995), diferenciaron el cambio a partir del nivel de análisis y lo clasificaron en dos tipos, a saber: continuo y discontinuo. El primero, también denominado de primer orden se da en un sistema estable y equilibrado. El segundo o de segundo orden representa una ruptura brusca del sistema y por ende genera transformaciones en la esencia de la organización. 
García y Forero (2015), clasifican los cambios según su propósito/objetivo, a saber:

1) Cambio estratégico: es un cambio impuesto por el entorno, tiene en cuenta factores económicos, sociales, políticos y legales, es la estrategia con la cual la empresa direccionará sus recursos para alcanzar sus objetivos; 2) Cambio institucional: implica cambios normativos y legales en la organización, que pueden incluir cambios en los reguladores de conducta hasta en los métodos para realizar funciones; 3) Cambio estructural: implica cambios en la estructura de la empresa, es decir, en los cargos, departamentos, personal y áreas de funcionamiento; 4) Cambio tecnológico: implica cambios en los productos, herramientas, procesos, técnicas implementados por la compañía; y el 5) Cambio cultural: hace referencia a un cambio a nivel de relaciones humanas en la institución como la visión, relaciones humanas, valores como el sentido de pertenencia, lealtad, las creencias, entre otros, que movilizan los trabajadores de una empresa a cumplir sus objetivos como comunidad.

Para Gutiérrez (2019), los cambios se pueden dar de dos maneras: cambio prescriptivo y cambio constructivista. El prescriptivo tiene que ver con una "secuencia de eventos (...) determinados previamente" (p. 4) y que son fácilmente predecibles ya que obedecen a un patrón; se trata de pequeños cambios que sumados generan un cambio importante. El constructivista, por su parte, "hace referencia a una progresión que es construida y emerge mientras el proceso de cambio se despliega, generando cambios de segundo-orden, es decir cambios que rompen con los supuestos y marcos básicos utilizados hasta entonces" (p. 4).

Cuando algunos de los cambios mencionados se presentan dentro de la organización, puede darse resistencia por parte de los empleados. Quirant y Ortega (2006) plantean la resistencia al cambio como un conjunto de comportamientos mediante los cuales los empleados enfrentan, impiden o retardan un cambio en la organización que les genera sentimientos de inseguridad y amenaza hacia sus necesidades, esto porque, según García y Forero (2014), el cambio no es visto por los empleados como una acción positiva, pues la posición que toman frente a este es de pensar que la empresa ha desmejorado.

En acuerdo con García (2015) la resistencia al cambio es "el costo por cambio de tarea" (p. 43 ), es decir, el costo que tiene que asumir el empleado o la organización al enfrentar la implementación de un cambio, considerado como amenazante para su gestión laboral, caso en el que la adaptación surge como opción. La resistencia al cambio se puede identificar de dos formas, de tipo abierta, que es cuando el personal realiza manifestaciones, huelgas, disminución en la productividad, falta de participación y trabajo defectuoso y de tipo encubierta, que se manifiesta con renuncias, solicitudes de traslado, pérdida de motivación, altas tasas de accidentes y errores, demora en la entrega de trabajo y ausentismo laboral sin justa causa (Robbins, 2004). Cabe anotar en este punto que, no siempre la resistencia al cambio se debe presentar como algo negativo, puesto que, si esta no se genera, no se podría reconocer verdaderamente si el cambio que se está adaptando a la organización es el más acertado y necesario (Robbins y Judge, 2009). 
Además, para evitar las consecuencias negativas de la resistencia al cambio, se debe mantener informado al personal, por esta razón, la comunicación cumple un papel fundamental para la realización de los cambios organizacionales (Suárez, 2014), puesto que, cuando se mantiene al personal informado, se dará más fácil la adaptación a éste (Montealegre y Calderón, 2007). Así mismo, aceptar el cambio, por parte de los empleados, puede estar relacionado con las habilidades organizacionales y comunicativas de los directivos (Arras, Jáquez y Fierro, 2008).

Sin embargo es de considerar que en procesos de medición sobre iniciativas de cambio organizacional, realizados por Caicedo Prado, encontró que "mantener informado" al personal no es suficiente para mitigar la resistencia al cambio. En tal sentido considera que la información no es suficiente para lograr, en primera instancia, la identificación, es decir, que el cambio sea apreciado como algo necesario y justificado. Pero tampoco es suficiente para movilizar comportamientos de apoyo o respaldo, ya que muchas veces esa información explica el qué del cambio, pero no el por qué o el para qué, con lo cual hay un vacío sobre cuál es la visión de cambio propuesta. De allí que deba tenerse presente la gestión emocional del cambio, ya que allí se encuentra muchas veces el dinamizador para reforzar el compromiso.

Esa gestión emocional depende de aspectos como (1) las experiencias pasadas de los empleados en los procesos de cambio, (2) las relaciones con el jefe o líder de equipo, incluyendo la existencia de espacios de diálogo y retroalimentación y (3) la asociación entre cambio y beneficio, es decir, la valoración que se hace de los impactos positivos del cambio no solo para la empresa y sus clientes, sino también para los propios colaboradores.

Cuando hay una buena comunicación con los trabajadores, se presenta una adecuada adaptación (García, Arias y Gómez, 2013), lo que permite que la resistencia al cambio sea mínima (Montealegre y Calderón, 2007). En este sentido, la comunicación interna se convierte en un aspecto clave para la ejecución de nuevas formas laborales como lo es el teletrabajo, pues favorece el cambio necesario para consolidar un entorno y una cultura organizacional altamente competitiva y adaptativa (Muñoz, Pabón y Valencia, 2017).

Por este motivo Robbins y Judge (2009) afirman que tanto la educación como la comunicación disminuyen la resistencia al cambio, al combatir las consecuencias de una información y comunicación deficientes, pues no se da pie al rumor al aclarar hechos y malos entendidos, "la comunicación es de ayuda para 'vender' la necesidad de un cambio" ( $p$. 623).

Así, las organizaciones deben informar a los empleados por medio de mensajes formales e informales, las razones u objetivos por los cuales se debe implementar un cambio y, a partir de esto, brindar el conocimiento necesario y las habilidades que se requieren para hacerlo posible. En otras palabras, se debe capacitar al personal y motivar su apoyo mediante recompensas, ya sean de carácter económico, de reconocimiento, de ascenso, entre otros (Jaros, 2010). 


\section{Factores sociodemográficos}

En resultados hallados por Amado (2018) se expone la relevancia del papel de los factores sociodemográficos en las dinámicas organizacionales, pues, por ejemplo, los trabajadores que tienen un nivel educativo alto se ubican en cargos donde reciben un salario ajustado a sus labores, evidenciando índices más altos de satisfacción laboral, por ende, un mejor rendimiento y desempeño, al igual que un mayor grado de compromiso con la empresa.

Hernández, Gallarzo y Espinosa (2011) afirman que determinadas variables sociodemográficas como la formación, educación y edad de los empleados generan de manera individual una forma de asumir la vida de forma adaptativa y cómoda. Por esto, cuando el cambio es implementado, las personas deben modificar su comportamiento.

Carrillo, Solano, Martínez y Gómez (2013), realizaron un estudio en profesionales de la salud, encontrando mayor satisfacción con la vida laboral en personas mayores, esto puede ser porque, al contar con mayor experiencia laboral, esta población posee una visión más objetiva de las situaciones que generalmente los jóvenes valoran como insatisfactorias. Además, hallaron mayor satisfacción en las mujeres, este resultado lo fundamentan por la feminización de las ocupaciones que tiene el ámbito sanitario u otras profesiones afines como la enfermería.

Sin embargo, Martínez, Olmedo y García (2016), en una investigación de las diferencias en los estilos de liderazgo según el sexo en diez cooperativas Españolas, encontraron que no hay diferencias en los estilos de liderazgo, sino en los principios que guían esos estilos entre hombres y mujeres; las mujeres se interesan y son más empáticas con el entorno, con las necesidades de los trabajadores y con el desarrollo de sus competencias y habilidades, mientras que los hombres se interesan principalmente por la vinculación de socios. De otro lado, Salgado, Leria, Arcos, Pineda y González (2018) hallaron que las mujeres presentan un temor mayor a enfrentar el cambio en comparación con los hombres.

En otra investigación, Fernández, Crego y Alcover (2008), plantearon que, al estar próximos a la jubilación, las mujeres presentan mayor motivación para retirarse laboralmente de la organización que los hombres, debido a que, según estos autores, las mujeres logran alcanzar libertad y control frente a lo que les gusta y les genera placer, teniendo en cuenta que, son ellas, quienes asumen las labores domésticas y se encargan de los pormenores familiares.

En cuanto a la edad, a mayor número de años cumplidos laborando, menor es la motivación e interés por retirarse de la empresa, esto se debe a que los empleados con más de 56 años manifiestan que comenzarán a tener problemas en su salud y presiones organizacionales mediante su aproximación al retiro (Fernández, Crego y Alcover, 2008).

Por otra parte, se ha encontrado que las personas veteranas muestran un mayor compromiso con la empresa, adoptando sus valores como propios, en cambio, los jóvenes, por sus ideas individuales y por sus expectativas de vida, no los adhieren fácilmente considerando el trabajo como algo pasajero (Kónya, Matić y Pavlović, 2016). 
Sin embargo, a pesar de que las personas de más edad muestran un mayor compromiso en comparación con los jóvenes, Bilbao (1999) plantea que los empleados de edad avanzada perciben una amenaza, exigencia cognitiva de aprendizaje, crisis de autoeficacia y miedo al fracaso, en comparación con los jóvenes que, a pesar de no adherir los valores corporativos como propios, si tienen mayor adaptabilidad a los cambios.

En el entorno nacional, se ha señalado que las personas que están en edad madura ( 37 años promedio), se encuentran más satisfechos con su trabajo y, aunque son los jóvenes los que inician con mayor entusiasmo, su felicidad dura poco (Farné y Vergara, 2007). En esta línea, un estudio encontró que los adultos mayores de 51 años presentan un menor temor hacia el cambio (Salgado, Leria, Arcos, Pineda y González, 2018).

Algunos estudios han señalado diferencias respecto al nivel educativo, evidenciando que las personas con un nivel de formación más alto tienen mayor disposición con la organización, pues, al estar mejor calificadas, están más decididas y tienen más ambición por tener reconocimientos y éxitos empresariales (Kónya, Matić y Pavlović, 2016). Algo semejante fue encontrado en un reporte previo, donde se halló que los colaboradores, entre menor nivel de escolaridad, menor era su satisfacción y disposición laboral, por tener menos oportunidades de ser ascendidos, reconocidos y obtener mejores ingresos (Franek, Mohelská, Zubr, Bachmann y Sokolová, 2014).

Teniendo en cuenta los hallazgos anteriormente mencionados, se teje aquí la hipótesis que factores sociodemográficos como la edad, el sexo y el nivel de escolaridad, posiblemente se relacionan o marcan diferencias con la disposición al cambio, por tanto, el objetivo principal es determinar la relación de la disposición al cambio organizacional con factores sociodemográficos como edad y nivel de escolaridad, al igual que con la diferencia entre sexo masculino y femenino, en una organización de la ciudad de Pereira. 


\section{Metodología}

\subsection{Diseño y participantes}

El presente estudio fue de tipo cuantitativo - no experimental (Kerlinger y Lee, 2001) y se presentó partir de un muestreo no probabilístico en el que participaron 61 personas.

De acuerdo con las variables sociodemográficas, la tabla 1 muestra que el $52,5 \%$ de la población es de sexo masculino y el $47,5 \%$ corresponde al sexo femenino; con respecto al nivel de escolaridad los que puntúan más alto son los participantes de nivel educativo de postgrado con un $73,8 \%$, seguido de pregrado con $19,7 \%$, técnico/ tecnológico con $4,9 \%$ y secundaria con 1,6\%; en cuanto a la edad, la media es de 38 años cumplidos.

\section{Tabla 1. Factores sociodemográficos}

\begin{tabular}{|c|c|c|c|c|c|}
\hline & & & & Frecuencia & Porcentaje \\
\hline \multirow[t]{2}{*}{ Sexo } & \multicolumn{3}{|c|}{ Femenino } & 29 & 47,5 \\
\hline & \multicolumn{3}{|c|}{ Masculino } & 32 & 52,5 \\
\hline \multicolumn{6}{|l|}{ Nivel de } \\
\hline \multirow[t]{5}{*}{ Escolaridad } & \multicolumn{3}{|c|}{ Secundaria } & 1 & 1,6 \\
\hline & \multicolumn{3}{|c|}{ Técnico/Tecnológico } & 3 & 4,9 \\
\hline & \multicolumn{3}{|c|}{ Pregrado } & 12 & 19,7 \\
\hline & \multicolumn{3}{|c|}{ Postgrado } & 45 & 73,8 \\
\hline & Mínimo & Máximo & Media & \multicolumn{2}{|c|}{ Desviación estándar } \\
\hline Edad & 22 & 65 & 38 & \multicolumn{2}{|r|}{9,141} \\
\hline
\end{tabular}

Consideraciones éticas. Este estudio se soportó en las directrices de la Ley 1090 de 2006. Es considerada con riesgo bajo, fue aprobada por el comité de ética de la organización objeto de estudio, se tuvieron en cuenta los lineamientos de la resolución 8430 del Ministerio de Salud (1993), por lo que se entregó un consentimiento informado que los participantes diligenciaron antes del instrumento. De igual manera se obtuvo la autorización de la institución para realizar la investigación, previa firma de un acuerdo de confidencialidad que prohíbe mencionar su nombre.

\subsection{Instrumentos}

Para evaluar el cambio se utilizó el instrumento de Medición de Cambio Organizacional, desarrollado por García y Forero (2016), el cual cuenta con tres macro-dimensiones a saber: individual, grupal y organizacional, de las que se desprenden las siete sub-dimensiones que guían las preguntas del cuestionario tales como motivación, aceptación, comunicación, 
liderazgo, clima, conocimiento y cultura. Adicional a esto se tiene que la confiabilidad total de la prueba basada en el Alfa de Cronbach es de 0.86. La finalidad de este instrumento es, primero, conocer la disposición y preparación en la que se encuentra la organización y sus empleados al momento de gestionar un cambio y segundo, que a partir de los resultados se posibilita la proyección del cambio en términos de cómo podría ser desarrollado (García y Forero, 2016).

Para indagar por los factores sociodemográficos, las investigadoras crearon una ficha que contenía preguntas como 1. ¿Qué edad tiene? 2. ¿Cuál es su nivel de escolaridad? y 3. ¿Cuál es su sexo?

\subsection{Procedimiento}

En primera instancia, se envió la propuesta sobre la investigación a la organización objeto de estudio y se explicó la aplicación que se deseaba realizar, posterior a esto, a través de un Formulario de Google, se otorgó un consentimiento informado en donde se les aclaró a los participantes de qué constaba la investigación y cuáles eran sus reglamentos éticos, seguido, se les explicó a los colaboradores en qué consistía el instrumento de Medición del Cambio. Por último, la matriz de datos en Excel se trasladó al software SPSS v.20.

Desde allí, se calcularon medidas de tendencia central (media) y de dispersión (desviación estándar). La comprobación de hipótesis se empleó a través de la estadística no-paramétrica, pertinente por la distribución asimétrica de los datos, estimada a través del estadístico de Kolmogorov-Smirnov $(n>50)$. Las comparaciones de medianas se efectuaron a través de $U$ Mann-Whitney para muestras independientes (hombres vs mujeres). La prueba de significancia fue estimada con el valor $p<.05$. Los coeficientes de correlación se estimaron con $r$ de Pearson para la variable de edad y Rho de Sperman para el nivel de escolaridad.

\section{Resultados}

Los coeficientes de correlación entre la edad, el nivel de escolaridad y las dimensiones de disposición al cambio no mostraron relaciones (Tabla 2). Entre las dimensiones se observaron correlaciones significativas: Individual vs. Grupal $(r=.70)$, Individual vs. Organizacional $(r=.49)$, y grupal vs organizacional $(r=.81)$, todos los valores con un valor $p<.01$, lo que significa que existe una correlación entre las dimensiones del instrumento empleado en esta investigación 
Tabla 2. Coeficientes de correlación entre edad ( $r$ de Pearson), nivel de escolaridad (Rho Spearman) y las dimensiones y los factores de disposición al cambio

\begin{tabular}{|l|c|c|c|c|c|}
\multicolumn{1}{|c}{ Variable } & 1 & 2 & 3 & 4 & 5 \\
\hline 1.Escolaridad & 1 & .24 & .11 & -.09 & -.05 \\
\hline 2.Edad & & 1 & .06 & -.08 & .02 \\
\hline 3.Individual & & & 1 & $.70^{* *}$ & $.49^{* *}$ \\
\hline 4.Grupal & & & 1 & $.81^{* *}$ \\
\hline 5.Organizacional & & & & 1 \\
\hline
\end{tabular}

Nota: 3 - 4 - 5 factores de disposición organizacional . ${ }^{* *} p<.01$. Fuente: elaboración propia

Los datos descriptivos de las dimensiones según el sexo se observan en la tabla 3 y el gráfico 1. La dimensión mejor puntuada es la individual, seguida por la organizacional y la grupal, respectivamente. En la comparación de los promedios por género, en todos los puntajes, es superior el de las mujeres, únicamente la dimensión individual fue más alta en los hombres.

\section{Tabla 3. Descriptivos de las dimensiones y factores de disposición al cambio}

\begin{tabular}{lcccccc}
\multicolumn{1}{c}{ Variable } & \multicolumn{2}{c}{\begin{tabular}{c} 
Masculino \\
\multicolumn{1}{c}{$(n=32)$}
\end{tabular}} & \multicolumn{2}{c}{$\begin{array}{c}\text { Femenino } \\
(n=29)\end{array}$} & \multicolumn{2}{c}{$\begin{array}{c}\text { Todos } \\
(n=61)\end{array}$} \\
& $M$ & $D E$ & $M$ & $D E$ & $M$ & $D E$ \\
Dimensión Individual & 4.7 & 0.7 & 4.4 & 0.8 & 4.6 & 0.8 \\
Motivación & 4.4 & 0.9 & 4.2 & 1.0 & 4.3 & 1.0 \\
Aceptación & 5.2 & 0.5 & 4.9 & 0.7 & 5.0 & 0.6 \\
\hline Dimensión Grupal & 4.3 & 0.8 & 4.4 & 0.8 & 4.4 & 0.8 \\
Comunicación & 4.0 & 0.9 & 4.1 & 0.9 & 4.1 & 0.9 \\
Liderazgo & 4.6 & 0.7 & 4.7 & 0.7 & 4.6 & 0.7 \\
\hline Dimensión Organizacional & 4.4 & 0.8 & 4.7 & 0.8 & 4.5 & 0.8 \\
Clima & 4.8 & 0.6 & 4.8 & 1.0 & 4.8 & 0.8 \\
Conocimiento & 3.8 & 1.1 & 4.3 & 1.0 & 4.1 & 1.1 \\
Cultura & 4.5 & 1.0 & 4.8 & 0.9 & 4.6 & 0.9 \\
\hline
\end{tabular}

Fuente: elaboración propia

Gráfico 1

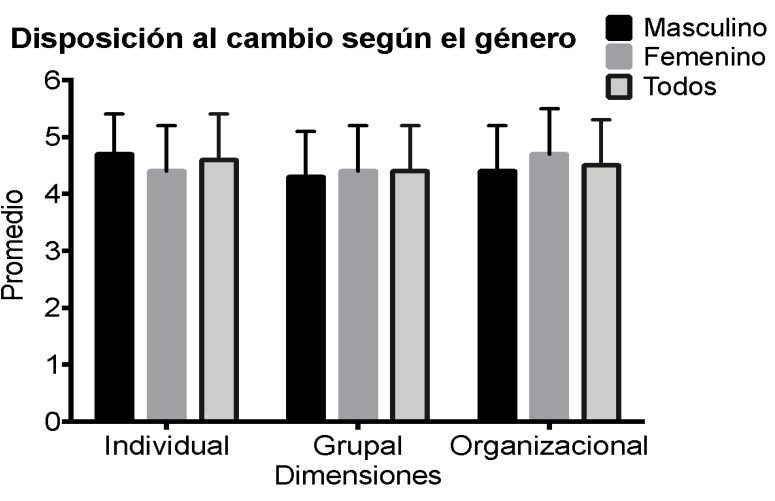

Fuente: elaboración propia 
En la tabla 4 se muestra el contraste de hipótesis a través del estadístico de U Mann Whitney. Los valores de mediana y rangos no mostraron diferencias significativas según el sexo $(p>.05)$, lo cual quiere señalar que el comportamiento de disposición al cambio es homogéneo entre hombre y mujeres.

Tabla 4. Contraste de hipótesis de las dimensiones

y los factores de disposición al cambio según el género o sexo

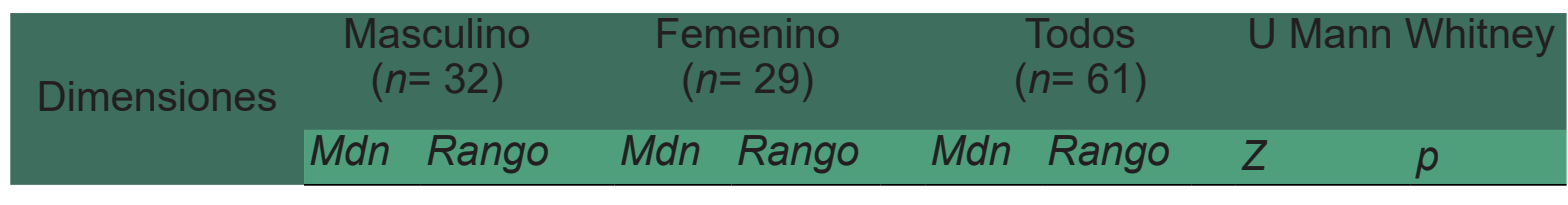

Individual
$4.7 \quad 2.7$
4.5
3.6
$4.7 \quad 3.9$
$-0.84$
.398

Grupal

\begin{tabular}{|c|c|c|c|}
\hline 4.1 & & & \\
\hline
\end{tabular}

Organizacional

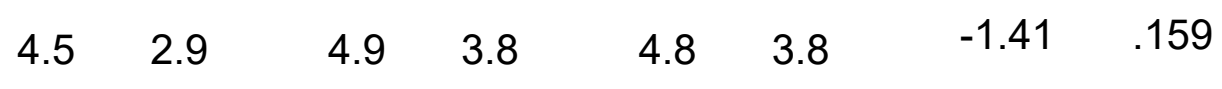

$p=$ disposición al cambio. Fuente: elaboración propia

\section{Conclusiones}

Con el objetivo de determinar la relación de la disposición al cambio organizacional con los factores sociodemográficos edad y nivel de escolaridad, al igual que con la diferencia entre sexo masculino y femenino, se encontraron dos hallazgos principales: (i) No hay relación entre la disposición al cambio organizacional con la edad y el nivel de escolaridad, (ii) Pese a que no se identificaron diferencias estadísticas entre la disposición al cambio y el sexo, sí se identificó una puntuación mayor en la dimensión individual para los hombres; mientras las mujeres obtuvieron una mayor puntuación en la dimensión grupal y en la organizacional.

Otro hallazgo importante se presenta en la dimensión individual-motivación y aceptación, el cual, obtuvo una mayor puntuación en comparación con las demás dimensiones. Se encontró que en la única dimensión donde las mujeres obtuvieron puntuaciones inferiores a los hombres fue en la dimensión individual, compuesta por factores de aceptación y motivación, esto tiene explicación desde Schaufeli y Enzmann (1998) quienes sostienen que el sexo femenino tiene rasgos en su personalidad que posibilitan el ajuste a los cambios, el control de eventos o capacidad social, más orientados hacia la actividad social. Esto permite inferir que las mujeres son más propensas a adaptarse al entorno, cuando lo hacen con la cooperación de otros que cuando lo hacen de manera individual.

De igual forma, las mujeres se interesan en mayor medida por la comunidad y el entorno social; muestran una mayor empatía con la toma de decisiones cuando es de manera grupal que cuando es individualizada (Martínez, Olmedo y García, 2016). Por esta razón, se 
considera que las mujeres son tendientes a comprender, de manera más abierta, los cambios y las necesidades que se pretenden implementar, para así, lograr una mejor reacción y adaptabilidad a los mismos, aunque los hombres se adaptan más fácil a los mismos de forma individual que en equipo. En este sentido, se tiene que ambos sexos cuentan con potencial para lograr asimilar o adaptarse a los cambios con recursos y estrategias diferentes entre sí.

Lo anterior, guarda coherencia con lo reportado por Salgado, Leria, Arcos, Pineda y González (2018), quienes afirman que las mujeres han obtenido una mayor puntuación frente a la disposición al cambio en comparación con los hombres, resaltando, igualmente, que la diferencia entre las puntuaciones no fue significativa. Es posible que no se hayan mostrado puntuaciones de gran relevancia debido a que los sujetos asimilan el cambio organizacional como algo recurrente en su entorno, lo que es de concluir que los participantes aceptan, de igual manera, todos los tipos de cambios.

En cuanto a la dimensión grupal, el liderazgo obtuvo la puntuación más alta $(M=4.6)$, cabe señalar que es el líder, la persona que guía y tiene una visión de futuro para orientar a sus colaboradores, y así, tener una mejor disposición frente al cambio. En este sentido, un estudio reciente señala la influencia del liderazgo transformacional sobre el compromiso afectivo hacia el cambio (Chou, 2019).

Por otro lado, en la dimensión grupal- comunicación, los sujetos presentaron una menor puntuación en comparación con las demás dimensiones y variables, lo que significa que los participantes consideran que tienen menos facilidades para emitir sus ideas y opiniones frente a los cambios organizacionales (García y Forero, 2010). En otro sentido, García, Arias y Gómez (2013) postulan que, una amplia comunicación y emisión de información no es suficiente si esta no es verídica o confiable para quien la recibe, se debe hacer partícipe al personal en la comprensión y colaboración de la ejecución del cambio desde la dirección de la organización.

Porret (2010) coincide en que el cambio implica un compromiso global de la organización, los objetivos de donde se quiere llegar deben ser definidos claramente, además, se deben brindar los conceptos, las competencias y demás que se requieran para ponerlo en práctica (Robbins, Decenzo y Coulter, 2009; Labarca, Ferrer y Villegas, 2006), como se evidenció en los resultados, una de las dimensiones donde se obtuvo puntuación baja fue en la organizacional, específicamente en conocimiento.

Cabe aclarar que, la dimensión mejor puntuada fue la individual, por ello se sugiere a la organización mantener y fortalecer tanto la aceptación como la motivación de sus trabajadores, así, puede ser de utilidad realizar un plan de gestión del cambio, donde se tenga en cuenta el desarrollo de competencias como el sentido de pertenencia y el compromiso con la organización, esto en pro de la creación de un vínculo fuerte entre la empresa y sus colaboradores e implicarlos en "la gestión de su propia empresa, es decir, de sus propios medios de producción" (León, 2016, p.5) con el fin de que participe un mayor número de personas, se logren resultados más precisos y se implementen las estrategias de cambio o gestión necesarias para el logro de sus propios procesos. Cabe resaltar que 
la preparación para el cambio debe tener como base los procesos formativos, donde se considere al talento humano como los promotores del mismo cambio, se debe tener en cuenta su opinión y su esfuerzo.

Una de las principales limitaciones de este estudio, estuvo relacionada con el tamaño de la muestra, pues se identificó una resistencia por parte de los trabajadores para responder el cuestionario, el cual, como se mencionó anteriormente contaba con una participación voluntaria.

Como indica Caicedo Prado, se debe considerar que hay otras variables demográficas que pueden tener influencia en la disposición del cambio y una de ellas es la antigüedad del colaborador, pero otra que también hemos identificado como determinante es el tipo de área en la que trabaja el colaborador. A manera de ejemplo, en una empresa $X$ encontramos que los colaboradores vinculados a un área encargada de la gestión de riesgos organizacionales estaban mucho menos dispuestos al cambio, en contraste con quienes trabajaban en áreas de investigación y desarrollo. También hemos identificado variaciones en los niveles de disposición al cambio según el tipo de cambio, pues en algunos casos los estratégicos generan menos resistencia que los tecnológicos y estructurales. Esto también puede estar asociado a las expectativas del colaborador: quienes tienen mayores pretensiones de permanecer por largo tiempo en la empresa pueden tener actitudes diferentes a quienes solo se ven de paso en la empresa. Son algunas de las posibilidades que se pueden abordar en futuras investigaciones.

Por último, resulta pertinente sugerir que futuros estudios puedan comparar este comportamiento psicosocial en organizaciones de diferentes sectores. Tener mayores avances investigativos en esta materia, será un insumo fundamental para diseñar planes de intervención que promuevan la disposición al cambio en los trabajadores.

\section{Referencias}

Acosta, C. (2002). Cuatro preguntas para iniciarse en cambio organizacional. Revista Colombiana de Psicología. (11), 9-24. En: http://www.redalyc.org/articulo.oa?id=80401101

Amado, M. (2018). Factores sociodemográficos, organizacionales y motivacionales asociados al desempeño laboral de los trabajadores de la Municipalidad Provincial de Huaylas - Caraz - 2016. (Tesis inédita de Maestría). Universidad Cesar Vallejo, Trujillo, Perú.

Arras, A., Jáquez, J. y Fierro, L. (2008). Comunicación y cambio organizacional. Revista Latina de comunicación social, 63, 418-434. Doi: https://doi.org/10.4185/RLCS-63-2008-792418-434

Carrillo, Solano, Martínez y Gómez (2013). Influencia del género y edad: satisfacción laboral de profesionales sanitarios. Revista Latino-Americana de Enfermagem, 21(6), 1314-1320. Doi: https://doi.org/10.1590/0104-1169.3224.2369

Chou, P. (2019). transformational leadership and attitude toward enterprise resource planning system: a perspective of organizational change. International Journal of 
Information Technology Project Management, 10 (1), 55-71. Doi: https://doi.org/10.4018/ IJITPM.2019010104

Fernández, J., Crego, A. y Alcover, C. M. (2008). Relaciones entre factores sociodemográficos, motivación hacia el retiro temprano y satisfacción en la vida postlaboral: análisis exploratorio en una muestra de prejubilados españoles. Revista de Psicología del Trabajo y de las Organizaciones. 24(3). 417-439. En: http://scielo.isciii.es/scielo. php?script=sci abstract\&pid=S1576-59622008000300008

Franek, M., Mohelská, H., Zubr, V., Bachmann, P. y Skolová, M. (2014). Organizational and Sociodemographic Determinants of Job Satisfaction in the Czech Republic. Sage. 1-12. En: http://journals.sagepub.com/doi/pdf/10.1177/2158244014552426

García, M. \& Forero, C. (2014). Motivación y satisfacción laboral como facilitadores del cambio organizacional: Una explicación desde las ecuaciones estructurales. En Psicogente, 17(31), 120-142. Doi: https://doi.org/10.17081/psico.17.31.1474

García, M. (2011). Liderazgo transformacional y la facilitación de la aceptación al cambio organizacional. Pensamiento psicológico, 9 (16), 41-54. En: http://www.redalyc.org/articulo. oa?id=80118612003

García, M. y Forero, C. (2010). IMC - Instrumento de Medición de Cambio Organizacional. Manual Técnico. Bogotá, Colombia: RH\&T.

García, M. y Forero, C. (2015). Contrato psicológico y cambio organizacional en una entidad perteneciente al sector terciario de la ciudad de Bogotá, Colombia. Rev.investig.desarro. innov, 6(1), 15-28. Doi: https://doi.org/10.19053/20278306.4047

García, M. y Forero, C. (2016). Calidad de vida laboral y la disposición al cambio organizacional en funcionarios de empresas de la ciudad de Bogotá-Colombia. Acta Colombiana de Psicología, 19(1), 79-90. Doi: https://doi.org/10.14718/ACP.2016.19.1.5

García, M., Arias, F. y Gómez, P. (2013). Relación entre comunicación y cambio organizacional en trabajadores de una empresa del sector terciario. Perspectiva psicológica, 9(1), 81-95. En: http://www.scielo.org.co/pdf/dpp/v9n1/v9n1a07.pdf

García, M., Rojas, M. y Díaz, S. (2011). Relación entre el cambio organizacional y la actitud al cambio en trabajadores de una empresa de Bogotá. Diversitas: Perspectivas en Psicología, 7(1), 125-142. En: http://www.redalyc.org/articulo.oa?id=67922583009

Garzón, M. (2005). El desarrollo organizacional y el cambio planeado. (1 ${ }^{a}$ ed). Bogotá: Centro editorial Universidad del Rosario.

Gutiérrez, R., V., Zambrano, J. \& Vásquez, J. (2019). Cambio organizacional, institucional y tecnológico: una aproximación desde la teoría actor-red y el trabajo institucional. Cuadernos de Administración. 32(59), 1-27. En: DOI: https://doi.org/10.11144/Javeriana.cao32-59.coit 
Hernández, J., Gallarzo, M., y Espinoza, J. (2011). Desarrollo organizacional: administración del proceso de desarrollo organizacional. México: Pearson.

Instituto Nacional de Estadística, Geografía e Informática (1997). Manual de medidas sociodemográficas. En:http://internet.contenidos.inegi.org.mx/contenidos/productos/prod serv/contenidos/espanol/bvinegi/productos/historicos/1329/702825000063/702825000063. pdf

Jaros, S. (2010). Commitment to Organizational Change: A Critical Review [El compromiso con el cambio organizacional: una revisión crítica]. Journal of Change Management, 10(1), 79-108. Doi: https://doi.org/10.1080/14697010903549457

Kerlinger, F. y Lee, H. (2001). Investigación del comportamiento. Métodos de investigación en ciencias sociales. ( $4^{\mathrm{a}}$ ed.) México: McGraw-Hill.

Kónya, V., Matić, D. y Pavlović, J. (2016). The Influence of Demographics, Job Characteristics and Characteristics of Organizations on Employee Commitment. [La influencia de las características demográficas, del trabajo y de las organizaciones en el compromiso de los empleados]. Acta Polytechnica Hungarica,13(3), 119-138. En: https://www.scirp.org/ $\underline{\text { (S(351jmbntvnsjt1aadkposzje))/reference/ReferencesPapers.aspx?ReferencelD }=2515600}$

Labarca, N., Ferrer, J. y Villegas, E. (2006). Cambio organizacional: Aspecto trascendental para las instituciones de educación superior en Venezuela. Revista de Ciencias Sociales, 12(1). 62-71. En: http://ve.scielo.org/scielo.php?script=sci abstract\&pid=S1315$95182006000100006 \&$ Ing=pt\&nrm=iso\&tlng=es

Ley 1090. Diario Oficial del CONGRESO DE LA REPÚBLICA No. 46.383 de 6 de septiembre de 2006. "Por la cual se reglamenta el ejercicio de la profesión de Psicología, se dicta el Código Deontológico y Bioético y otras disposiciones”. Bogotá, Colombia.

León, J. (2016). Importancia del sentido de pertenencia del trabajador en el funcionamiento de empresas autogestionadas. Facultad de psicología Universidad de la República de Uruguay. Recuperado de: https://www.academia.edu/30003826/lmportancia del sentido de pertenencia del trabajador en el funcionamiento de empresas autogestionadas 1

Maldonado, I., Maldonado, M. y Bustamante, S. (2006). Clima organizacional y gerencia: Inductores del cambio organizacional. (Tesis inédita de Maestría). 21(2). 231-248. En: http://www.redalyc.org/articulo.oa? id=65821209

Martínez, I., Olmedo, I., y García, L. (2016). Diferencias de género en el estilo de liderazgo en las cooperativas de enseñanza. Universidad Politécnico de Cartagena, España. En: http:// ciriec.es/wp-content/uploads/2016/10/COMUN-172-T11-MARTINEZ-OLMEDO-GARCIA-ok. $\underline{\mathrm{pdf}}$

Ministerio de Salud (1993). Resolución No. 8430 de 1993. Por la cual se establecen las normas científicas, técnicas y administrativas para la investigación en salud. Ministerio de Salud. Gobierno de Colombia. En: https://www.minsalud.gov.co/sites/rid/Lists/ 
Montealegre, J., \& Calderón, G. (2007). Relaciones entre actitud hacia el cambio y cultura organizacional. Estudio de caso en medianas y grandes empresas de confecciones de Ibagué. Innovar, 17(29), 49-70. En: https://revistas.unal.edu.co/index.php/innovar/article/ view/19585/20634

Muñoz, D., Pabón, M. y Valencia, B. (2017). Comunicación organizacional entorno al teletrabajo. Revista Luciérnaga - Comunicación, 9 (18), 61-71. En: https://revistas.elpoli.edu. co/index.php/luc/article/view/1256

Nadler, D. y Tushman, M. (1995). In Discontinuous Change: Leading Organizational Transformation. San Francisco: Jossey-Bass.

Porret, M. (2010). Gestión de personas: Manual para la gestión del capital humano en las organizaciones. (4a ed.). Madrid: ESIC Editorial.

Quirant, A. y Ortega, A. (2006). El cambio organizacional: La importancia del factor humano para lograr el éxito del proceso de cambio. Revista de empresa, 18, 50-63. En: https://dialnet.unirioja.es/servlet/articulo?codigo=2240827

Raeder, S. (2019). The role of human resource management practices in managing organizational change. Gruppe. Interaktion. Organisation, 50, 169-191. https://doi. org/10.1007/s11612-019-00465-1

Robbins, S., Decenzo, D., A., Y Coulter, M. (2009). Fundamentos de la administración. (8a ed.) México: Pearson. En: https://www.academia.edu/28751366/Fundamentos de administracion - Stephen P. Robbins.pdf

Robbins, S \& Judge (2009). Comportamiento Organizacional. (13 ed.) México: Pearson Educación.

Robbins, S. (2004). Comportamiento Organizacional: Resistencia al cambio. (10 ed.). México: Pearson Prentice Hall.

Salgado, J., Leria, F., Arcos, L., Pineda, C. y González, C. (2018). Actitud y resistencia al cambio organizacional en trabajadores mineros. Revista de psicología, 36 (1), 105-134. Doi: https://doi.org/10.18800/psico.201801.004

Segredo, A. (2013). Clima organizacional en la gestión del cambio para el desarrollo de la organización. Revista cubana de salud pública, 39(2). 385-393. En: http://scielo.sld.cu/scielo. php?script=sci arttext\&pid=S0864-34662013000200017

Schaufeli, W y Enzmann, D. (1998). El compañero de burnout para estudiar y practicar: un análisis crítico. Filadelfia: Taylor y Francis.

Suárez, M. (2014). Determinación de la relación entre la comunicación y el liderazgo en la aceptación del cambio organizacional en un ambiente universitario en la ciudad de Bogotá. 
(Tesis de maestría). Universidad Católica de Colombia, Bogotá D.C, Colombia.

Valle Flórez, M. M. (2016). La Investigación en Comunicación Organizacional en Antioquia - Colombia. Luciérnaga Comunicación, 7(13), 55-65. https://doi.org/10.33571/ revistaluciernaga.v7n13a4

\section{Para citar este artículo}

Castaño, E., Quiroz, E., Pulgarín, L., Britto, V. (2020). Cambio organizacional: análisis de factores sociodemográficos. Revista Luciérnaga Comunicación. Vol. 12 Núm. 24. Pp: 71-89 https://doi.org/:10.33571/revistaluciernaga.v12n24a5

OJS: https://revistas.elpoli.edu.co/index.php/luc/issue/archive

Link:_https://www.politecnicojic.edu.co/index.php/revista-luciernaga 